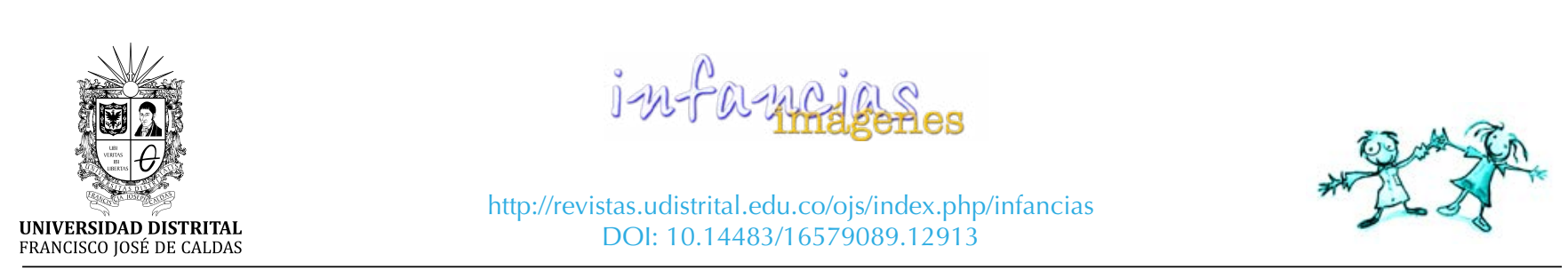

IMÁGENES DE INVESTIGACIÓN

\title{
Sabores y saberes en la escuela: experiencia de intervención educativa*
}

\section{Flavors and knowledge at school: educational intervention experience}

\author{
Alejandra María Rodríguez Guarín", Sandra Faisuler Potosí Rodríguez², \\ Mabel Cristina Calvache Muñoz ${ }^{3}$, Salomón Rodríguez Guarín ${ }^{4}$
}

Para citar este artículo: Rodríguez, A. M.; Potosí, S. F.; CalRecibido: 29-diciembre-2017 / Aprobado: 16-noviembre-2018 vache, M.C.; Rodríguez, S. (2018). Sabores y saberes en la escuela: experiencia de intervención educativa. Infancias Imágenes, 18(1), 36-50

\section{Resumen}

Investigación descriptiva y de corte etnográfico, a partir de la estrategia "Sembrando vida para crecer sanos", dirigida a escolares de dos instituciones educativas de la ciudad de Popayán, Cauca (Colombia). En su implementación se analizaron dos tensiones presentes en la configuración de los hábitos alimentarios de los escolares producto de la interacción de los saberes-aprendizajes de la familia, y los gestados en otros escenarios de relacionamiento en la escuela, como la tienda/restaurante escolar o el recreo, junto con narrativas y significaciones del alimento. Entre sus resultados, se encuentra la generación de diálogos de saberes alrededor de prácticas alimentarias cotidianas, revitalizadas en la escuela como institucionalidad legitimada para la formación de hábitos o costumbres.

\begin{abstract}
Se destaca la construcción de didácticas para fomentar el consumo de frutas y verduras, y la integración de conceptos como: gestión del territorio, soberanía-seguridad alimentaria y nutrición a los $\mathrm{PEI}^{5}$ de las instituciones.

Palabras clave: escolar, alimentación escolar, pedagogía experimental, hábitos alimentarios, frutas y verduras.
\end{abstract}

\section{Abstract \\ Descriptive and ethnographic research, based on the strategy "Sowing Life to Grow Healthy", aimed at schoolchildren from two educational institutions in the city of Popayan, Cauca (Co- lombia). In its implementation, two present matters were analyzed in the configuration of students' food habits as a result of the interaction between the knowledge-learning family, and}

* Artículo de investigación derivado de los proyectos "Estrategia multicomponente para incrementar el consumo de frutas y verduras en estudiantes de la I.E. Los Comuneros de Popayán-Cauca" y "Estrategia multicomponente para incrementar el consumo de frutas y verduras en estudiantes en edad escolar (6 a 12 años) del colegio técnico Comfacauca de Popayán-Cauca. Desarrollados durante los años 2014 a 2017.

1 Estudiante de Doctorado en Antropología, Magister en Estudios Interdisciplinarios del Desarrollo, Ingeniera de Alimentos, Líder del grupo de investigación Investigarte Unicomfacauca, Corporación Universitaria Comfacauca - Unicomfacauca, Popayán (Colombia). Correo electrónico: investigarte@unicomfacauca.edu.co

2 Magister en Ciencias Químicas, Química, Directora del programa Tecnología en Gestión Gastronómica, docente investigadora del grupo de investigación Investigarte Unicomfacauca. Corporación Universitaria Comfacauca - Unicomfacauca. Popayán (Colombia). Correo electrónico: sfpotosi@unicomfacauca.edu.co

3 Magister en Ingeniería de Alimentos, Ingeniera Agroindustrial, Docente Investigadora del grupo de investigación Investigarte Unicomfacauca. Corporación Universitaria Comfacauca - Unicomfacauca. Popayán (Colombia). Correo electrónico: mcalvache@unicomfacauca.edu.co

4 Magister en Filosofía, Licenciado en Filosofía, Decano Facultad de Humanidades, Artes, Ciencias Sociales y de la Educación, Docente Investigador del grupo de investigación Investigarte Unicomfacauca. Corporación Universitaria Comfacauca - Unicomfacauca. Popayán (Colombia). Correo electrónico: decanatura@unicomfacauca.edu.co

5 Proyecto Educativo Institucional. 
those generated in other relationship settings at the school, such as the school store/restaurant or recess, together with narratives and significances of food. Among its results, is the generation of dialogs of knowledge around daily food practices, revitalized in the school as institutions legitimized for the formation of habits or customs. The building of teaching tools to promote the consumption of fruits and vegetables stands out, as well as the integration of concepts such as: territorial management, food security-sovereignty and nutrition for the PEI of the institutions.

Keywords: schoolchildren, school feeding, educational experiments, food habits, fruits and vegetables.

\section{Introducción}

Los alimentos son fundamentales para la supervivencia y bienestar social. Con tal propósito, desde temprana edad, las comunidades han diseñado y puesto en marcha diversas estrategias culturales para interiorizar o canalizar ${ }^{6}$ la ingesta de un determinado alimento, así como para reafirmar imaginarios asociados a las raciones y formas de consumo (Rodríguez y Rodríguez, 2017). Por su parte, Moscovici (1979) considera que la alimentación constituye un aspecto ligado a las formas y manifestaciones de pensamiento social, al incluir informaciones, creencias, actitudes, prácticas o experiencias, las cuales han denominado representaciones sociales; en suma, estas proveen contenidos significativos a los comportamientos y hábitos. De acuerdo con Jodelet (1989), dichas representaciones podrían aportar en el diseño de intervenciones como, por ejemplo: programas nutricionales y educacionales, en virtud de la influencia que ejercen sobre la conducta alimentaria y el impacto esperado de los mismos.

Los argumentos de Moscovici (1979) son susceptibles de otorgar nuevas dimensiones a las investigaciones destinadas a identificar los factores que causan problemáticas de desnutrición, especialmente en la población infantil; condición reconocida en 1992 durante la Cumbre Mundial de la Alimentación, considerado como un fenómeno que

6 Canalización/interiorización: este concepto se asocia, en la práctica, a la configuración de hábitos, costumbres y formas de consumo a partir de una instrucción previa, que se construye, en la mayoría de los casos, desde algunos agenciamientos como la escuela, la familia, el mercado entre otros, que pueden ser asimilados como "un proceso psicológico por medio del cual, las necesidades tienden a hacerse más específicas como consecuencia de haber sido satisfechas en forma específica"... en tanto, "lo arbitrario se vuelve natural" (Bock, 1977). pone en riesgo el buen desarrollo de la humanidad. Es de resaltar que en este escenario muchos gobiernos se comprometieron a promover estudios de esta naturaleza y a utilizarlos en la formulación de políticas en alimentación y nutrición (FAO, 1992; citada por Rodríguez y Rodríguez, 2017).

Particularmente, Lowenberg, Wilson, Todhunter, Feeney y Savage (1970) afirman que las políticas alrededor del tema alimentario deben considerar a la alimentación como un acto revestido de factores sociales y psicológicos, mediado por un sistema complejo de actitudes, ideas, presupuestos, religión o preceptos de tipo moral, que en la mayoría de los casos se interiorizan desde los primeros años de vida.

\section{Cifiras y realidades}

En la elección alimentaria, influyen al igual que en las representaciones sociales gestadas desde la niñez los imaginarios o las creencias acerca de su elección y las políticas que median su acción, la regularidad en los ingresos, su forma de distribución, los medios de conservación, la información sobre el valor nutricional, los agenciamientos como el mercado y los medios de comunicación, entre otros (Rodríguez y Rodríguez, 2017). Así mismo, en la mayoría de los países de Latinoamérica y el Caribe, la alimentación se ha caracterizado, además, por la baja proporción de alimentos de origen vegetal, así como el alto contenido de grasas saturadas de origen animal, sal y azúcares refinadas (Rodríguez y Rodríguez, 2017); estos, en muchas ocasiones, no aportan los nutrientes necesarios para mantener un sano desarrollo y conservar una buena salud, esencialmente en la edad escolar (6 a 12 años).

Equiparable a ello, se encuentra la influencia de la economía y la cultura globalizada. A partir de 
allí se conjugan otros aspectos estrechamente relacionados con acciones y políticas internacionales como, por ejemplo, el reconocimiento del artículo 25 de la Declaración Universal de los Derechos Humanos, que hace referencia a que: "toda persona tiene derecho a un nivel de vida adecuado que le asegure, así como a su familia, la salud y el bienestar, y en especial la alimentación...", acciones de las Naciones Unidas para la Alimentación y la Agricultura (FAO, 2015) o del Programa Mundial de Alimentos (2007).

En el caso del departamento del Cauca, tomando en cuenta las mediciones realizadas por el Departamento Administrativo Nacional de Estadística en Colombia (Dane), este presenta un índice de necesidades básicas insatisfechas (NBI) de 46.62 $\%$, siendo del $24.27 \%$ en cabeceras municipales y del $61.97 \%$ en zonas rurales (Dane, 2011); en el mismo contexto, la incidencia de pobreza extrema en 2014 fue de $24 \%$ y el coeficiente de Gini de 0.535 (Dane, 2014) $^{7}$. En contraste, su riqueza en términos de biodiversidad y cultura lo convierten en un territorio de alta complejidad, en el cual desarrollan sus vidas comunidades con identidades y conflictividades diversas (Corporación Autónoma Regional del Cauca, 2009), en las que se ubica la tenencia de la tierra, junto con la obtención de alimentos en cantidad y calidad suficiente en el primer reglón. En relación con este aspecto, es de anotar que el Cauca se encuentra entre los cinco territorios con los índices más altos de desnutrición global, cifra que disiente de su riqueza y diversidad, presentando indicadores significativos en torno a situaciones de obesidad y sobrepeso (Secretaría de Salud Departamental del Cauca, 2017). Algunos de estos descriptores se resumen a continuación:

Frente al eje del consumo, articulada con las problemáticas detectadas, se encuentra la promoción de la alimentación y los estilos de vida saludable, pensada como una política nacional en las instituciones educativas (básica primaria y secundaria), la cual trata de contrarrestar las condiciones de subnutrición que presentan muchos de los niños y niñas inscritos al sistema educativo. En términos globales, pretende lograr el incremento en la distribución e ingesta de alimentos de alto valor nutricional, como son las frutas y las verduras, al ser una fuente importante de fibra, carotenoides, licopeno, ligninas, ácidos fenólicos y compuestos azufrados, vitamina $\mathrm{A}$, ácido fólico, vitamina $\mathrm{C}$, tiamina, riboflavina y ácido nicotínico, los cuales tienen efectos positivos en la salud. En un ámbito biológico, "el consumo diario de al menos tres a cinco porciones de frutas y verduras pueden inhibir o retrasar la aparición de enfermedades crónicas, debido a su capacidad para modular los procesos biológicos a través de sus nutrientes y compuestos fitoquímicos" (Crujeiras, 2010). Además, según la Organización Mundial de la Salud (2007), el bajo consumo de verduras y frutas origina más del $30 \%$ de enfermedades de corazón en el mundo.

Tabla 1. Resumen de las problemáticas frente al estado de seguridad alimentaria y nutricional en el departamento del Cauca.

\begin{tabular}{|c|c|c|c|c|}
\hline $\begin{array}{l}\text { Acceso a los } \\
\text { alimentos }\end{array}$ & $\begin{array}{c}\text { Disponibilidad de los } \\
\text { alimentos }\end{array}$ & $\begin{array}{c}\text { Aprovechamiento } \\
\text { biológico }\end{array}$ & Consumo & $\begin{array}{l}\text { Calidad e } \\
\text { inocuidad }\end{array}$ \\
\hline $\begin{array}{l}\text { Pérdida de la capa- } \\
\text { cidad productiva de } \\
\text { los territorios y de } \\
\text { las comunidades. } \\
\text { Inadecuadas prác- } \\
\text { ticas agrícolas y uti- } \\
\text { lización deficiente } \\
\text { de los recursos } \\
\text { naturales. }\end{array}$ & $\begin{array}{l}\text { Falta de ingresos económicos por } \\
\text { parte de familias vulnerables, para } \\
\text { adquirir alimentos saludables. } \\
\text { Aumento en los precios de los } \\
\text { alimentos saludables debido a la } \\
\text { pérdida de cosechas como con- } \\
\text { secuencia del cambio climático. } \\
\text { Inadecuadas rutas de comerciali- } \\
\text { zación de alimentos saludables. }\end{array}$ & $\begin{array}{l}\text { Baja cobertura } \\
\text { de saneamiento } \\
\text { básico, calidad de } \\
\text { agua y manejo de } \\
\text { vectores. } \\
\text { Baja cobertura de } \\
\text { servicios sociales y } \\
\text { de salud. }\end{array}$ & $\begin{array}{l}\text { Falta de educación de cuidadores } \\
\text { y padres/madres, respecto al valor } \\
\text { nutricional de los alimentos. } \\
\text { Creencias e inadecuadas prácticas } \\
\text { alimenticias. } \\
\text { Incorporación de dietas inadecua- } \\
\text { das producto de la moda. } \\
\text { Incorporación de nuevas dietas, ge- } \\
\text { neradas por la pérdida de patrones/ } \\
\text { hábitos alimenticios tradicionales. }\end{array}$ & $\begin{array}{l}\text { Deficiente } \\
\text { inspección } \\
\text { y vigilancia } \\
\text { para hacer } \\
\text { cumplir la } \\
\text { normativi- } \\
\text { dad. }\end{array}$ \\
\hline
\end{tabular}

Fuente: Secretaría de Salud Departamental del Cauca (2017).

7 Datos que lo ubican como uno de los departamentos con el mayor índice de necesidades básicas en Colombia, al lado del Chocó que históricamente es el más desigual en ingresos y calidad de vida. 
Tabla 2. Resumen de acciones para enfrentar los indicadores seguridad alimentaria y nutricional en el departamento del Cauca, en articulación con lo establecido por la FAO.

\begin{tabular}{|c|c|c|c|c|}
\hline $\begin{array}{l}\text { Acceso a los } \\
\text { alimentos }\end{array}$ & $\begin{array}{l}\text { Disponibilidad de } \\
\text { los alimentos }\end{array}$ & $\begin{array}{c}\text { Aprovechamiento } \\
\text { biológico }\end{array}$ & Consumo & $\begin{array}{l}\text { Calidad e } \\
\text { inocuidad }\end{array}$ \\
\hline $\begin{array}{l}\text { Producción sostenible y } \\
\text { amigable de alimentos } \\
\text { con enfoque territorial y } \\
\text { uso adecuado de recur- } \\
\text { sos naturales. }\end{array}$ & $\begin{array}{l}\text { Familias vulnerables } \\
\text { con acceso a alimen- } \\
\text { tos nutritivos (huertas } \\
\text { caseras). }\end{array}$ & $\begin{array}{l}\text { Población habitan- } \\
\text { do territorios con } \\
\text { saneamiento básico, } \\
\text { servicios de salud y } \\
\text { manejo de vectores. }\end{array}$ & $\begin{array}{l}\text { Recuperación de prácticas de } \\
\text { alimentación tradicional. } \\
\text { Incorporación a la vida cotidia- } \\
\text { na de estilos de vida saludables. } \\
\text { Aumento de consumo alimentos } \\
\text { no procesados. }\end{array}$ & $\begin{array}{l}\text { Inspección y vigi- } \\
\text { lancia para hacer } \\
\text { cumplir la normati- } \\
\text { vidad. }\end{array}$ \\
\hline
\end{tabular}

Fuente: Secretaría de Salud Departamental del Cauca (2017).

En este sentido, las prácticas y los elementos que enmarcan las dinámicas alrededor de los alimentos podrían ser susceptibles de transformaciones y adaptaciones, al estar entrelazadas con condicionamientos esencialmente humanos. De no ser abordadas adecuadamente, pueden conducir a tensiones e incluso a la reconfiguración de concepciones o preceptos frente al territorio, cuyo impacto dependerá del manejo y las intervenciones realizadas en cada comunidad en particular. Esta sintomatología no es ajena a la población de la institución educativa (IE) Los Comuneros y el colegio técnico Comfacauca (ubicadas en la ciudad de Popayán, Cauca), cuya población en los dos casos presenta, en términos generales, tanto condiciones de subnutrición como de sobrealimentación.

\section{Inicio del camino}

Basados en descriptores, la investigación se llevó a cabo tomando en cuenta los siguientes momentos:

En primer lugar, la exploración inicial de la población escolar en las dos IE puso de manifiesto la necesidad de establecer procesos de intervención hilvanando de forma efectiva el ejercicio del concepto seguridad alimentaria ${ }^{8}$, tratando de incrementar el consumo de frutas y verduras de la región, así como su abordaje desde los elementos que ofrece la soberanía alimentaria ${ }^{9}$, siendo la escuela un es-

8 De acuerdo con la FAO, el concepto hace referencia, a que todas las personas deberán tener en todo momento acceso físico, social y económico a los alimentos en cantidad y calidad suficiente; Estos deben satisfacer las necesidades biológicas y energéticas diarias, y las preferencias alimentarias con el fin de llevar una vida sana y activa (2011).

9 En términos generales, hace referencia al derecho de los pueblos a definir sus propias políticas y estrategias sustentables de producción, comercialización y consumo de alimentos que garanticen el pacio ideal para que los niños y niñas en edad escolar (6 a 12 años) reconozcan las bondades de su territorio en materia alimentaria, identifiquen los nutrientes asociados a sus prácticas alimentarias o interioricen hábitos adecuados desde la niñez (Rodríguez y Rodríguez, 2017), al tiempo que evocan saberes y sabores, compendios que hacen parte del inventario cultural de una región.

En segundo lugar, a partir de la caracterización de la población, se aplicó una estrategia de intervención multicomponente ${ }^{10}$ denominada: "Sembrando vida para crecer sanos". De manera general, la estrategia pretendió brindar a los integrantes de las comunidades académicas:

Elementos para el desarrollo de acciones que fortalezcan el ejercicio de la transformación social, todo ello, con base en las características socioeconómicas y culturales que han demarcado los procesos implementados en materia de seguridad y soberanía alimentaria dispuestos en sus proyectos educativos institucionales y en su hacer cotidiano, al tiempo que busca generar su empoderamiento es decir, estimular por autodescubrimiento, el incremento en el consumo frutas y verduras, y el fomento de una alimentación adecuada que supla los requerimientos nutricionales de los niños(as) y de sus familias, haciendo uso de perspectivas de enseñanza-aprendizaje participativas e incluyentes. (Rodríguez y Rodríguez, 2017, p.163)

derecho a la alimentación para toda la población con base en la pequeña y mediana producción, respetando sus propias culturas y diversidad (Foro Mundial Sobre Soberanía Alimentaria celebrado en la Habana, Cuba; septiembre de 2001).

10 Multicomponente: involucra escolares, directivos, padres/madres de familia, profesores y manipulares de alimentos de las tiendas $y$ restaurante escolar de las IE. 
Los problemas de malnutrición en los últimos años se han incrementado a nivel global, bien sea por las limitaciones en el acceso a los alimentos o por los inadecuados hábitos nutricionales como el bajo consumo de frutas y verduras. Así, es como se genera la necesidad de crear estrategias pedagógicas para la resignificación de una alimentación saludable, nutritiva y agradable, respetando las costumbres y las culturas de las diferentes comunidades. En este sentido, los momentos metodológicos aplicados permitieron, además de develar los actuales hábitos y estilos culturales de alimentación urbana a partir del análisis de la población escolar de dos IE, validar la implementación de estrategias de corte multicomponente. Esto con el ánimo de propiciar el desarrollo de habilidades comunicativas, destrezas motrices, hábitos alimenticios saludables, fomentando con ello estilos de vida saludables, tomando como referencia, que la malnutrición por exceso de azúcar o el uso de azucares refinados, deficiencia en la ingesta de vitaminas y minerales desde las frutas y verduras, entre otras, principalmente en la población infantil urbana de la nación se está convirtiendo en un problema de salud pública.

En este orden de ideas, el presente artículo pretende evidenciar la relación existente entre cifras, realidades y acciones dinamizadas por las IE, así como la necesidad de involucrar procesos de enseñanza-aprendizaje que permitan mejorar sus costumbres alimenticias, calidad de vida y sus condiciones nutricionales, sin perder de vista los saberes propios de cada comunidad.

\section{Metodología}

\section{Tipo de investigación}

La investigación retomó los elementos que ofrece el tipo exploratorio-descriptivo (Hernández Fernández y Baptista, 2006), con el propósito de asociar las experiencias como punto de partida para el reconocimiento y valoración de prácticas alimentarias permeadas en muchos aspectos por dinámicas de intercambio de saberes y sabores, junto con la presión del impacto en los esquemas dietarios a partir del posicionamiento de comidas preparadas, y el posicionamiento hoy con mayor fuerza de cargas de estatus social a través del consumo, alejado del atributo simbólico que implica el acto de alimentarse.

Se utilizaron, además, algunos preceptos de la etnografía en virtud de develar desde la voz de sus actores sus preocupaciones sobre el tema, así como afianzar lazos de confianza con la población objeto de estudio (escolares de 6 a 12 años).

La recolección de la información se realizó durante los años 2014 y 2017, en las instalaciones de la IE Los Comuneros y el colegio técnico Comfacauca de la ciudad de Popayán, Cauca (Colombia). Su desarrollo se hizo a partir de tres fases, explicadas en la figura 1:

Figura 1. Estructura temática y metodológica de la estrategia multicomponente para incrementar el consumo de frutas y verduras en los escolares de las IE.

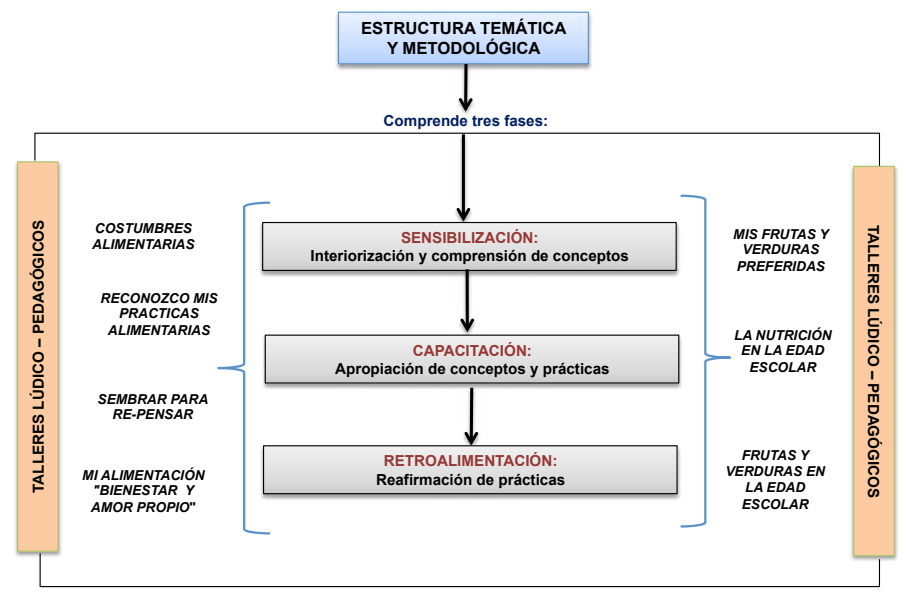

Fuente: elaboración propia de los autores. 


\section{Fuentes y procedimientos para recolección de} información

En las diferentes fases, se recurrió a fuentes de información primaria y secundaria, así como el diseño e implementación de técnicas de recolección y análisis de información de tipo cualitativo y cuantitativo. En el primer caso, se usaron los siguientes instrumentos metodológicos:

- Entrevista semi-estructurada. Se constituyó en el mecanismo de aproximación hacia el reconocimiento de las prácticas asociadas al consumo de frutas y verduras por parte de los escolares de 6 a 12 años, sus padres de familia, los docentes, directivos docentes y manipuladores del restaurante y tienda escolar que se involucran en el proceso. Las preguntas que se manejaron fueron de tipo cerrado y abierto, combinadas con preguntas de reflexión en el caso de los padres de familia, docentes y directivos docentes, y de interpretación para los estudiantes.

- Grupos focales (GF) con estudiantes, padres de familia y docentes. Este proceso se realizó mediante la constitución de grupos focales (GF), en los cuales intervinieron en conjunto y por separado escolares, padres de familia, docentes, directivos docentes y personal integrado al restaurante y tienda escolar, teniendo en cuenta la muestra considerada para el estudio. El criterio para establecer el número máximo de integrantes fue el rango de edad, máximo 20 y mínimo 10 personas, a quienes se les proporcionaron las orientaciones necesarias, las cuales permitieron el desarrollo de diversos talleres bajo un enfoque lúdico y pedagógico.

- Talleres de cartografía con los escolares, padres de familia y manipuladoras de alimentos. Se concibe como una herramienta de planificación y transformación social, que permite, grosso modo, la construcción del conocimiento a partir de la participación y el compromiso de un determinado grupo humano, posibilitando el reconocimiento de valores y procesos culturales, incluso de relevos generacionales. Su utilización fue muy valiosa para el diagnóstico de las prácticas culturales asociadas a las identidades alimentarias de la comunidad académica, con énfasis en la utilización de frutas y verduras, así como en la construcción de nuevas significaciones alrededor de su práctica alimentaria, en sus dimensiones de salud, nutrición y educación.

- Talleres de sensibilización y capacitación, orientados a fomentar el consumo de frutas y verduras, con énfasis en el rescate de productos locales. Este material se construyó con base en los resultados de los instrumentos usados durante las diferentes fases del proyecto, con énfasis en la etapa de sensibilización.

- En el mismo orden de importancia, se recurrió al uso de recursos audiovisuales como fotografías e información adicional suministrada por los encargados de los procesos de manipulación en los restaurantes escolares, y entidades relacionadas con el tema. Las fuentes secundarias se constituyeron en la revisión documental acorde al tema abordado: bases de datos, bibliografía especializada, videos, entre otros. El análisis estadístico de los datos se llevó a cabo con Microsoft Excel 2010.

\section{Población y muestra}

La población estuvo constituida por 289 niños y niñas de la IE Los Comuneros y por 242 niños(as) del colegio técnico Comfacauca, de los grados $1^{\circ}$, $2^{\circ}, 3^{\circ}, 4^{\circ}$ y $5^{\circ}$ de básica primaria matriculados entre los años 2014 a 2017 con edades que oscilan entre los 6 a los 14 años, inscritos en la jornada de la mañana (tabla 3). La muestra final quedó conformada por aquellos estudiantes que tuvieron la autorización formal de sus padres para participar en las actividades programadas (tabla 4). Durante

Tabla 3. Población objeto de estudio.

\begin{tabular}{|l|c|c|c|c|c|}
\hline \multirow{2}{*}{\multicolumn{1}{c}{ Institución Educativa }} & \multicolumn{4}{c}{ Población considerada } \\
\cline { 2 - 6 } & Estudiantes & $\begin{array}{c}\text { Padres/madres } \\
\text { de familia }\end{array}$ & \multicolumn{1}{c}{ Profesores(as) } & \multicolumn{1}{c}{ Manipuladores(as) } & $\begin{array}{c}\text { Total } \\
\text { población }\end{array}$ \\
\hline IE Los Comuneros & 280 & 280 & 10 & 2 & 320 \\
\hline Colegio técnico Comfacauca & 186 & 186 & 11 & 5 & 244 \\
\hline
\end{tabular}

Fuente: elaboración propia de los autores. 
todo el proceso participaron niños y niñas de ambos géneros, inscritos en la jornada de la mañana.

\section{Resultados y discusión}

\section{Escolares, realidades y formas de consumo}

A continuación, se presentan los datos obtenidos por medio la encuesta semiestructurada; su implementación permitió caracterizar algunos de los elementos necesarios para la implementación de la estrategia de intervención educativa. Los escolares que participaron (tabla 5), fueron a $57.6 \%$ de niñas y $42.4 \%$ de niños.

Frente a la fuente principal de ingresos y su núcleo familiar (tabla 6), se pudo establecer que la mayoría de las familias de los niños(as) pertenecientes al colegio técnico Comfacauca contaban con trabajos estables, en que el padre y la madre trabajaban; igualmente, están constituidos en su núcleo familiar principalmente por su padre y madre con un $(36.36 \%)$ y en segundo lugar su padre, madre y hermanos (34.34\%). Caso contrario sucede en la IE Los Comuneros, donde las familias no cuentan, en su gran mayoría, con un trabajo estable, por lo que se dedican a actividades informales, con un alto porcentaje de personas en condición de vulnerabilidad y algunas incluso desplazadas de zonas aledañas a la ciudad de Popayán; su núcleo familiar está constituido en primer lugar por padre y madre con un $(68 \%)$ y en segundo lugar por padre, madre y abuelos con un $(20 \%)$.

Tabla 4. Muestra objeto de estudio.

\begin{tabular}{|l|c|c|c|c|c|}
\hline \multirow{1}{*}{ Institución Educativa } & \multicolumn{5}{c}{ Muestra considerada } \\
\cline { 2 - 6 } \multicolumn{1}{c|}{} & Estudiantes & $\begin{array}{c}\text { Padres/madres } \\
\text { de familia }\end{array}$ & \multicolumn{1}{c}{ Profesores(as) } & Manipuladores(as) & $\begin{array}{c}\text { Total } \\
\text { población }\end{array}$ \\
\hline IE Los Comuneros & 50 & 28 & 10 & 2 & 90 \\
\hline Colegio técnico Comfacauca & 99 & 42 & 11 & 5 & 157 \\
\hline
\end{tabular}

Fuente: elaboración propia de los autores.

Tabla 5. Estudiantes en edad escolar de las IE.

\begin{tabular}{|c|c|c|c|c|c|}
\hline \multirow{2}{*}{ Institución Educativa } & \multicolumn{5}{|c|}{ Género de los escolares } \\
\hline & Estudiantes & Niñas & Niños & $\%$ Niñas & \% Niños \\
\hline IE Los Comuneros & 50 & 33 & 17 & 66 & 34 \\
\hline Colegio técnico Comfacauca & 99 & 57 & 42 & 57.6 & 42.4 \\
\hline
\end{tabular}

Fuente: elaboración propia de los autores.

Tabla 6. Con quién viven los escolares encuestados

\begin{tabular}{|c|c|c|c|c|}
\hline \multirow{2}{*}{ ¿Con quién vives? } & \multicolumn{2}{|c|}{ Colegio técnico Comfacauca } & \multicolumn{2}{|c|}{ IE Los Comuneros } \\
\hline & Total & Porcentaje & Total & Porcentaje \\
\hline Papá y mamá & 36 & 36.36 & 34 & 68 \\
\hline Papá y mamá, hermanos & 34 & 34.34 & - & 0.0 \\
\hline Mamá & - & - & 6 & 12 \\
\hline Papá y mamá, abuelos & 6 & 6.06 & 10 & 20 \\
\hline Papá, mamá, hermanos, tíos y abuelos & 6 & 6.06 & - & \\
\hline Papá, mamá, hermanos y abuelos & 6 & 6.06 & - & \\
\hline Papá y mamá, tíos & 2 & 2.02 & - & \\
\hline Abuelos & 2 & 2.02 & - & \\
\hline Abuelos, hermanos & 2 & 2.02 & - & \\
\hline Papá, mamá, hermanos y tíos & 2 & 2.02 & - & \\
\hline Papá, mamá, abuelos y tíos & 2 & 2.02 & - & \\
\hline Mamá y hermano & 1 & 1.02 & - & \\
\hline Total escolares encuestados & 99 & 100 & 50 & 100 \\
\hline
\end{tabular}

Fuente: elaboración propia de los autores. 
Frente a la preparación de los alimentos (tabla 7), los escolares del colegio técnico Comfacauca afirman en un alto porcentaje que los alimentos son preparados por el padre o la madre $(67.68 \%)$, al igual que los escolares de la IE Los Comuneros.

A pesar de que en la mayoría de los casos los padres/madres les preparan los alimentos, hay que tener en cuenta que a medida que los niños(as) crecen y acceden a nuevos contextos de socialización, aparecen otras fuentes que coadyuvan al desarrollo de sus funciones de comunicación, comprensión, adquisición de normas. Al mismo tiempo, irán asimilando y adquiriendo valores, que en su conjunto le permitirán consolidar o modificar aspectos de su desarrollo sociopersonal (Savater, 1997).
En un ámbito general, los escolares consideran que el hogar o la familia nuclear sigue siendo su espacio propicio para recibir sus alimentos, lo cual se traduce para ellos(as) en afecto o manifestaciones de cariño. Así mismo, lo asumen como un territorio en el que además de continuar interiorizando prácticas que replica en otros espacios de socialización, le ayudarán a resignificar las prácticas heredadas de sus padres, madres, abuelos o tíos, siendo estas representaciones ampliamente fijadas en este espacio social (Jodelet, 1989).

Sobre las elecciones alimentarias, Ios escolares de la IE Los Comuneros prefieren los alimentos con alto valor proteico, entre los que sobresalen la leche, los huevos, y en menor proporción la carne, así como los carbohidratos (figura 2); en menor

Tabla 7. Quién prepara los alimentos de los escolares encuestados.

\begin{tabular}{|c|c|c|c|c|}
\hline \multirow{2}{*}{ ¿Quién te prepara tus alimentos? } & \multicolumn{2}{|c|}{ Colegio técnico Comfacauca } & \multicolumn{2}{|c|}{ IE Los Comuneros } \\
\hline & Total & Porcentaje & Total & Porcentaje \\
\hline Papá y mamá & 67 & 67.68 & 33 & 66 \\
\hline Abuelos(a) & 8 & 8.08 & 15 & 30 \\
\hline Mamá & 0 & 0.0 & 2 & 4 \\
\hline Papá y mamá, hermanos & 6 & 6.06 & 0 & 0.0 \\
\hline Papá y mamá, abuelos & 5 & 5.05 & 0 & 0.0 \\
\hline Papá y mamá, tíos & 4 & 4.04 & 0 & 0.0 \\
\hline Papá, mamá, abuelos y tíos & 3 & 3.03 & 0 & 0.0 \\
\hline Papá, mamá, hermanos y abuelos & 1 & 1.01 & 0 & 0.0 \\
\hline Tíos & 1 & 1.01 & 0 & 0.0 \\
\hline Hermanos & 1 & 1.01 & 0 & 0.0 \\
\hline Papá, mamá, hermanos, tíos y abuelos & 1 & 1.01 & 0 & 0.0 \\
\hline Otros, ¿quiénes? & 2 & 2.02 & 0 & 0.0 \\
\hline Total escolares encuestados & 99 & 100 & 50 & 100 \\
\hline
\end{tabular}

Fuente: elaboración propia de los autores.

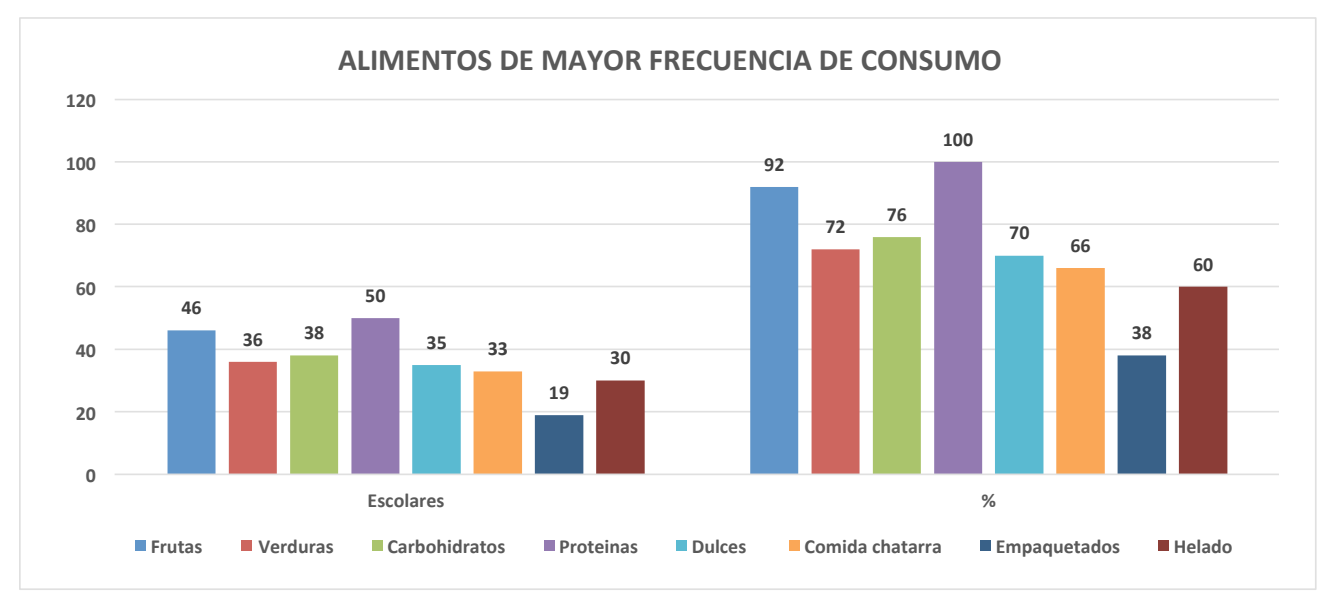

Figura 2. Encuesta de escolares IE Los Comuneros - Consumo de alimentos.

Fuente: elaboración propia de los autores. 
proporción se encuentran las frutas y verduras. Lo anterior se establece como un descriptor de la dieta en la nación, concentrada en los cereales, raíces, tubérculos y plátanos, grupo alimentario que aporta cerca de la mitad de la energía diaria, una parte las proteínas, y frutas y verduras en último lugar (Ministerio de Salud y Protección Social, 2015).

En la etapa escolar las comidas adquieren un significado social importante, al existir gran influencia de los amigos, los medios de comunicación y el mercado, sobre su elección alimentaria (Rodríguez y Rodríguez, 2017). Es entendible al respecto que los escolares de la IE Los Comuneros prefieran consumir sus alimentos en la casa con sus padres/madres y en segundo lugar en el recreo de su tienda escolar con sus amigos. Igualmente, los padres/madres o cuidadores desempeñan un papel importante en la transferencia de los hábitos alimentarios al tener la capacidad de incidir en las porciones y tipos de productos que ellos(as) deberán consumir.

Por su parte, de los escolares del colegio técnico Comfacauca se puede afirmar que el consumo de alimentos proteicos (pollo, carne, huevos, leche y pescado) es de aproximadamente el $26.9 \%$ (tabla 8), comportamiento atribuible, en gran medida, a su mayor capacidad de ingresos; sin embargo, se observa nuevamente el bajo consumo de frutas y verduras.

Tabla 8. Encuesta de escolares colegio técnico Comfacauca, Consumo de alimentos.

\begin{tabular}{|c|c|c|c|}
\hline \multirow{2}{*}{\multicolumn{2}{|c|}{ ¿Alimentos que consumen con mayor frecuencia? }} & \multicolumn{2}{|c|}{ Colegio técnico Comfacauca } \\
\hline & & Total & Porcentaje \\
\hline \multirow{5}{*}{ Proteína } & Pollo & 36 & \multirow{5}{*}{26.90} \\
\hline & Carne & 21 & \\
\hline & Huevos & 17 & \\
\hline & Leche y derivados & 35 & \\
\hline & Pescado & 23 & \\
\hline \multirow{6}{*}{ Frutas } & Manzana & 29 & \multirow{6}{*}{24.13} \\
\hline & Naranja & 27 & \\
\hline & Fresa & 19 & \\
\hline & Sandia & 6 & \\
\hline & Pera & 21 & \\
\hline & Uvas & 16 & \\
\hline \multirow{4}{*}{ Verduras y tubérculos } & Zanahoria & 15 & \multirow{4}{*}{12.10} \\
\hline & Tomate & 15 & \\
\hline & Plátanos & 23 & \\
\hline & Papa & 6 & \\
\hline \multirow{6}{*}{ Granos } & Choclo & 17 & \multirow{6}{*}{11.40} \\
\hline & Frijol & 8 & \\
\hline & Arroz & 16 & \\
\hline & Avena & 0 & \\
\hline & Lenteja & 5 & \\
\hline & Arveja & 10 & \\
\hline \multirow{5}{*}{$\begin{array}{l}\text { Dulces y paquetes } \\
\text { (alimentos azucarados y } \\
\text { almidones) }\end{array}$} & Bombones & 20 & \multirow{5}{*}{19.02} \\
\hline & Chocolates & 30 & \\
\hline & Helado & 22 & \\
\hline & Galletas & 12 & \\
\hline & Papas en paquete & 9 & \\
\hline \multirow{3}{*}{ Comidas rápidas } & Perros & 12 & \multirow{3}{*}{6.34} \\
\hline & Hamburguesas & 10 & \\
\hline & Chorizos & 9 & \\
\hline
\end{tabular}

Fuente: elaboración propia de los autores. 
La tabla anterior representa el comportamiento de los escolares frente al consumo de productos empaquetados como bombones, chocolates, helados, galletas o papas fritas. Una posible explicación a esta tendencia de consumo puede atribuirse al placer que le otorga -en el sentido del gusto interactúan la lengua y el paladar, las papilas gustativas que detectan el sabor dulce se encuentran en la punta de la lengua-.

Con respecto a las comidas rápidas, los escolares del colegio técnico Comfacauca aproximadamente al $6.34 \%$ eligen perros calientes, hamburguesas y chorizos, alimentos con una fuente calórica elevada. Su elección puede deberse al nivel de ingresos de sus padres, así como la presión de los medios de comunicación, en virtud del impacto que genera en la mente del escolar (Muñoz, 2004). El consumo de frutas y verduras en los escolares del colegio técnico Comfacauca corresponde a un $36.23 \%$ como se observa en la tabla 8, esta información es de gran importancia debido a su valor nutricional. Estos compuestos pueden ser importantes en la prevención de enfermedades relacionadas con la dieta, ya sea solo o, en algunos casos, en combinación con los frutos secos y otros alimentos vegetales (Crujeriras, Goyenechea y Martínez, 2010; Pennington y Fisher, 2009).

\section{Descubriendo saberes y sabores: estrategia} multicomponente para incrementar el consumo de frutas y verduras en los escolares de las IE A continuación, se describen los principales resultados de las fases puestas en marcha durante la investigación:

\section{Fase de sensibilización (interiorización y comprensión de} conceptos)

El propósito de este momento metodológico fue lograr que los participantes comprendieran los conceptos vinculados a su práctica alimentaria cotidiana; esta etapa estuvo soportada con elementos de la cartografía social y la observación de los participantes. Su desarrollo requirió de la constitución de diferentes grupos focales en los cuales intervinieron en conjunto y por separado estudiantes, padres de familia, docentes y directivos docentes, manipuladores de alimentos, teniendo en cuenta la muestra considerada para el estudio. Con el uso de la lúdica, se develaron las posibilidades desde sus saberes para fomentar el consumo de frutas y verduras. Inicialmente se trabajó con la comunidad educativa: los profesores(as), los directivos(as) docentes, manipuladores(as) de alimentos del restaurante escolar y padres/madres de familia (tabla 9).

Algunos de los aspectos a resaltar durante esta fase, fueron:

\section{Costumbres alimentarias}

Con el desarrollo de esta actividad se logró conocer acerca de las costumbres alimentarias de los padres-madres de familia, que a su vez son transmitidas a sus hijos(as) y, en general a su entorno familiar. Con el diálogo generado entre padres y capacitadores se logró establecer que muchos de los alimentos que ellos consumían en su infancia como frutas y verduras han dejado de ser parte de su canasta familiar. De acuerdo con lo expresado por los participantes, esto se debe a la situación socioeconómica actual pues, en algunas ocasiones, no cuentan con el dinero necesario para comprarlos. Así mismo, manifiestan que deben salir a trabajar en busca de su sustento, por lo cual sus hijos deben quedar al cuidado de otras personas, y en el peor de los casos solos o al cuidado de hermanos mayores. Es de resaltar que, al momento de plasmar la información en el material entregado, el consumo de otro tipo de alimentos en su niñez, donde se hace evidente la ingesta de frutas y verduras en mayor proporción, mientras que en la actualidad se ha incrementado el consumo de comidas rápidas, o de fácil adquisición como: perros, salchipapas (papa y salchicha), pizza, carnes procesadas o embutidos, alimentos empaquetados, entre otros.

Otro factor a tener en cuenta, es el impacto de los medios de comunicación, sumado al mismo entorno en el que viven; en muchas ocasiones, estas variables generan impacto poco asertivo en las dietas tradicionales. Los niños(as), y a la vez sus padres, en muchas ocasiones por comodidad han comenzado a interiorizar gustos, hábitos y preferencias por encima de las frutas y las verduras, dejando claro, que muchos de los comportamientos individuales se hallan influenciados por variables del entorno, como son los factores culturales, 
Tabla 9. Actividades de sensibilización.

\begin{tabular}{|c|c|c|}
\hline Actividad & Objetivo General & Descripción de la actividad/metodología \\
\hline $\begin{array}{l}\text { Taller 1. Costum- } \\
\text { bres alimentarias }\end{array}$ & $\begin{array}{l}\text { Identificar los procesos de sobera- } \\
\text { nía alimentaria que mantienen los } \\
\text { padres/madres de familia de los } \\
\text { niños(as) de las IE. }\end{array}$ & $\begin{array}{l}\text { Taller lúdico pedagógico, utilizando la técnica de la cartografía social, } \\
\text { cuyo objetivo es reconocer a través del diálogo el sentir de sus costum- } \\
\text { bres, en virtud de procesos de soberanía alimentaria. Taller realizado } \\
\text { para padres/madres de familia de los grados tercero, cuarto y quinto. }\end{array}$ \\
\hline $\begin{array}{l}\text { Taller } 2 \text {. Recono- } \\
\text { cimiento de mis } \\
\text { prácticas alimen- } \\
\text { tarias }\end{array}$ & $\begin{array}{l}\text { Identificar y reconocer los hábitos } \\
\text { alimenticios que tienen los ni- } \\
\text { ños(as) de las IE. }\end{array}$ & $\begin{array}{l}\text { Taller lúdico pedagógico, usado para reconocer las prácticas alimen- } \\
\text { tarias asociadas al entorno familiar a la vez que permite realizar un } \\
\text { diagnóstico acera de las costumbres alimentarias de los niños. Fue el } \\
\text { primer acercamiento con los (las) escolares. Taller realizado con los } \\
\text { niños(as) de quinto de primaria. }\end{array}$ \\
\hline $\begin{array}{l}\text { Taller } 3 . \\
\text { Mis frutas y ver- } \\
\text { duras preferidas }\end{array}$ & $\begin{array}{l}\text { Identificar las particularidades y } \\
\text { preferencias de los niños(as) esco- } \\
\text { lares de las IE. }\end{array}$ & $\begin{array}{l}\text { Taller lúdico pedagógico, para lograr que los niños reconozcan a partir } \\
\text { del juego sus frutas y verduras preferidas que se perciben en sus imagi- } \\
\text { narios, asociadas a las prácticas alimentarias al entorno familiar; Taller } \\
\text { realizado con los niños(as) de segundo de primaria. }\end{array}$ \\
\hline $\begin{array}{l}\text { Taller } 4 . \\
\text { Mi alimentación } \\
\text { "Bienestar y amor } \\
\text { propio" }\end{array}$ & $\begin{array}{l}\text { Implementación de actividades } \\
\text { que conduzcan a través del juego } \\
\text { a reconocer la importancia de la } \\
\text { alimentación saludable y el con- } \\
\text { sumo de frutas y verduras para el } \\
\text { crecimiento de los niños(as). }\end{array}$ & $\begin{array}{l}\text { Taller lúdico pedagógico usado para lograr que reconozcan a partir } \\
\text { del juego la importancia de una alimentación saludable y como ele- } \\
\text { mento esencial el consumo, uso de las frutas y verduras en su alimen- } \\
\text { tación diaria. Taller realizado con los niños(as) de primero y cuarto de } \\
\text { primaria. }\end{array}$ \\
\hline $\begin{array}{l}\text { Taller } 5 . \\
\text { Sembrar para } \\
\text { alimentarse y } \\
\text { re-pensar }\end{array}$ & $\begin{array}{l}\text { Desarrollar huertos escolares } \\
\text { como plataforma de aprendiza- } \\
\text { je para lograr una alimentación } \\
\text { saludable. }\end{array}$ & $\begin{array}{l}\text { Taller lúdico pedagógico, se buscó identificar las características y } \\
\text { potencialidades de la huerta casera, para el mejoramiento de las } \\
\text { condiciones nutricionales de la comunidad. Taller realizado con los } \\
\text { niños(as) de segundo de primaria. }\end{array}$ \\
\hline
\end{tabular}

Fuente: elaboración propia de los autores.

sociales, económicos y geográficos. En dicho entramado sociocultural, la interacción gestada entre las poblaciones humanas y su alimentación, surgida a partir de costumbres que con el uso se vuelven características cotidianas propias (Marín et al., 2004) $y$, con el pasar del tiempo, pueden sufrir modificaciones por factores ajenos a la misma cultura, ello requerirá entonces de procesos de interpretación desde el diálogo de saberes y sabores.

\section{Reconocimiento de mis prácticas alimentarias}

Esta actividad se realizó con el fin de tener un primer acercamiento con los escolares, al tiempo que permitió realizar un diagnóstico frente a sus costumbres alimentarias. Sus resultados permitieron establecer que el consumo de frutas y verduras se encuentra en un lugar alejado de su dieta. Los escolares denotan alta preferencia por alimentos ricos en azucares, e incluso de fácil preparación, en correspondencia a lo manifestado por los padres/ madres de familia en el primer taller realizado. Se corrobora que la elección y consumo de un alimento es el resultado, en primer lugar, de prácticas traídas de su familia y, en segundo lugar, en la escuela. Lo anterior se constituye en una oportunidad para las IE en su modelo pedagógico e incluso en sus didácticas para la enseñanza-aprendizaje de aspectos nutricionales.

\section{Mi alimentación: "Bienestar y amor propio"}

A través de juego se invitó a los escolares de los grados primero y cuarto a participar en diferentes actividades cuyo objetivo fue interiorizar conceptos y reconocer la importancia del amor propio. En tal caso, se presentaron varias frutas y verduras a partir de ejercicios lúdicos, en los que se utilizó la danza y la música. Por ejemplo, en el caso de las fresas, se les manifestó que se consumen directamente en ensaladas de frutas o para salpicón, provienen del campo, son producidas por campesinos, aportan nutrientes como la vitamina $C$, vitamina $A$, vitamina $E$, potasio y magnesio, entre otras propiedades. En general, los niños(as) se mostraron atentos a la explicación; sin embargo, se percibió que algunos(as) tienen una baja concentración, y se distraen con facilidad. Del mismo modo, cuando la orientadora le preguntaba a algún niño para qué se utilizaba la fruta o verdura, cuál era su procedencia o aporte nutricional, a los estudiantes de primer año les quedaba muy difícil responder, lo cual se 

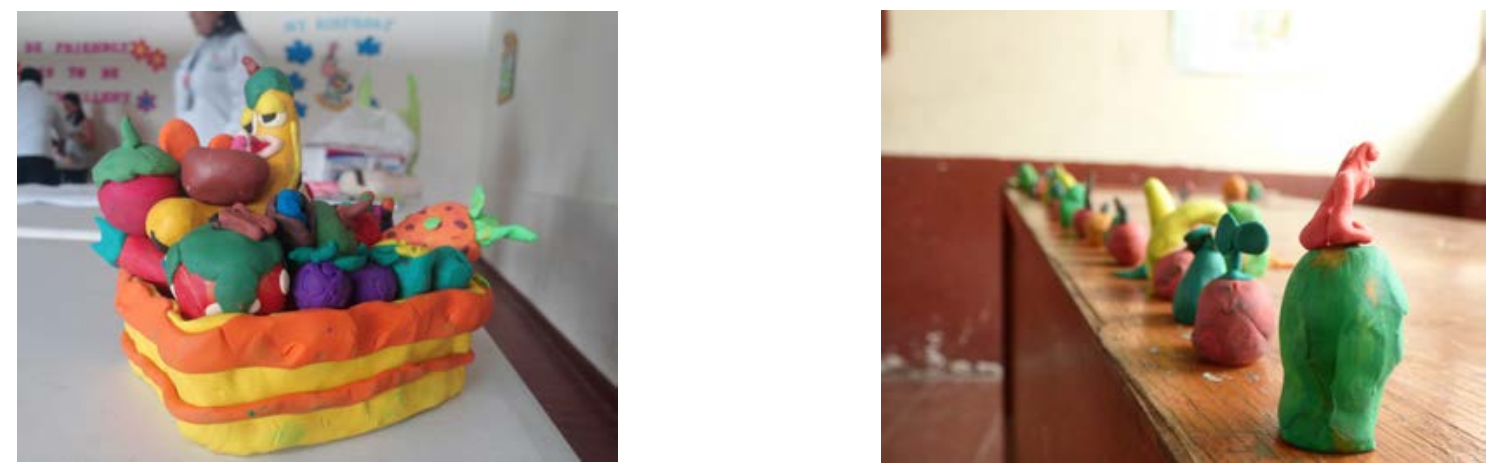

Figura 3. Taller frutas y verduras de mi región.

Fuente: elaboración propia de los autores.

debe en gran parte a su corta edad y construcción de conceptos.

\section{Sembrar para alimentarse y re-pensar}

A través del taller teórico-práctico se invitó a estudiantes del grado segundo a recocer la importancia del huerto escolar de su institución, socializando conceptos como el respeto y amor a la naturaleza. El taller se diseñó de manera interdisciplinaria, fortaleciendo los temas relacionados con la alimentación, la nutrición y la soberanía alimentaria con base en el nivel, grado y capacidad de percepción de los escolares.

Fase de capacitación (apropiación de conceptos y prácticas) A partir del análisis de los resultados obtenidos en el trabajo de campo, se pusieron en marcha acciones de aula cuyo objetivo principal fue estimular el rol educativo con base en el desarrollo cognoscitivo, tomando como referencia algunos de los preceptos de Piaget (1972): los métodos pedagógicos deben apelar a la actividad espontánea es decir, su función no es de impartir conocimientos sino de crear un ambiente que estimule la iniciativa, el juego, la experimentación, el razonamiento y colaboración social (tabla 10).

El desarrollo de los contenidos estuvo destinado a plantear las características de la sana alimentación en edad escolar, con el fin de trabajar en la escuela sobre los hábitos alimentarios de la población infantil, además de revisar el papel de los comedores escolares y las normas de higiene alimentaria. Mediante los contenidos y las actividades desarrolladas se desarrollaron estrategias didácticas con el objetivo de contribuir al mejoramiento de algunos de los problemas nutricionales presentes en la comunidad académica.

El desarrollo de esta fase permitió develar que el ambiente dentro de la escuela fomenta el aumento en el consumo de alimentos altamente energéticos, los cuales aportan $46 \%$ de la energía, al tiempo que limitan el acceso a agua potable, frutas y verduras (Delgado y Benavides, 2012), siendo indispensable el uso de acciones concretas para contrarrestar dicha dinámica. La escuela también constituye uno de los principales entornos de formación, internalización y creación cultural, en tanto que es reconocida como un ambiente ideal para generar cambios en conductas enfocadas a estilos de vida saludables o, en este caso, lograr una educación efectiva en nutrición, siendo indispensable interiorizar conceptos a partir de ejercicios que llamen la atención y contribuyan a motivar el cambio en los niños y niñas de edad escolar.

\section{Fase de retroalimentación (reafirmación de prácticas)}

Durante esta fase, a partir de diversas actividades realizadas previamente, la comunidad académica logró reconocer saberes y sabores vinculados con su alimentación, es decir, contenidos nutricionales, formas de consumo, texturas y evocaciones de la niñez. Esto, reencontrándose con sus prácticas tradicionales, toda vez que se buscaba consolidar procesos sostenibles para mejorar la condición nutricional de los niños(as) y las personas a su cuidado; para tal fin, se desarrollaron las siguientes actividades (tabla 11). 
Tabla 10. Actividades desarrolladas en la fase de capacitación.

\begin{tabular}{|c|c|c|}
\hline Actividad & Objetivo General & Descripción de la actividad/metodología \\
\hline $\begin{array}{l}\text { Charlas informa- } \\
\text { tivas: } \\
\text { alimentación salu- } \\
\text { dable y nutrición }\end{array}$ & $\begin{array}{l}\text { Concientizar a la comunidad educa- } \\
\text { tiva de la importancia que tiene para } \\
\text { la salud la práctica de buenos hábitos } \\
\text { alimentarios. }\end{array}$ & $\begin{array}{l}\text { Charla de corte teórico, cuyo objetivo fue mostrar a los profesores } \\
\text { y profesoras de las IE la importancia que tiene una buena alimen- } \\
\text { tación en los niños(as) en edad escolar. } \\
\text { Para ello socializaron los nutrientes esenciales que aportan los } \\
\text { alimentos y su relación con la salud, la nutrición y la soberanía } \\
\text { alimentaria, buscando de esta manera mejorar las condiciones de } \\
\text { alimentación de los escolares y el reconocimiento del potencial } \\
\text { culinario y gastronómico de la región. Taller realizado para profe- } \\
\text { sores(as) de básica primaria de las IE. }\end{array}$ \\
\hline $\begin{array}{l}\text { Charlas informa- } \\
\text { tivas: la nutrición } \\
\text { en la edad escolar }\end{array}$ & $\begin{array}{l}\text { Capacitar a los padres/madres de fa- } \\
\text { milia en cultura, alimentación escolar } \\
\text { y los aspectos nutricionales de las } \\
\text { frutas y verduras. } \\
\text { Construir y enseñar preparaciones sa- } \\
\text { ludables con base de frutas y verduras } \\
\text { de la región. }\end{array}$ & $\begin{array}{l}\text { Charla de corte teórico-práctico, en la cual se fortalecieron los } \\
\text { conceptos relacionados con seguridad, soberanía alimentaria } \\
\text { y nutrición, además de dotar de fundamentos prácticos para } \\
\text { elaborar alimentos saludables que contribuyan al desarrollo y la } \\
\text { formación de los niños(as). } \\
\text { Taller realizado con madres y padres de familia, manipulado- } \\
\text { res(as) y profesores(as) de las IE. }\end{array}$ \\
\hline $\begin{array}{l}\text { Charlas informa- } \\
\text { tivas: } \\
\text { frutas y verduras } \\
\text { en la edad escolar }\end{array}$ & $\begin{array}{l}\text { Desarrollar un espacio de reconoci- } \\
\text { miento sobre la importancia de la ali- } \\
\text { mentación adecuada desde temprana } \\
\text { edad, y cómo diversificar el consu- } \\
\text { mo de frutas y verduras; construir y } \\
\text { enseñar preparaciones saludables con } \\
\text { base de frutas y verduras de la región. }\end{array}$ & $\begin{array}{l}\text { Charla de corte teórico-práctico, en la cual se dialogó acerca del } \\
\text { valor cultural y nutricional de los alimentos, consolidando en ellos } \\
\text { una actitud de autocuidado a través de las prácticas alimentarias } \\
\text { saludables, además de fortalecer su rescate y valoración, sumado } \\
\text { a fomentar prácticas amigables y sustentables con el ambiente. } \\
\text { Taller realizado con escolares, madres y padres de familia y mani- } \\
\text { puladores(as). }\end{array}$ \\
\hline
\end{tabular}

Fuente: elaboración propia de los autores.

Tabla 11. Actividades desarrolladas en la fase de retroalimentación.

\begin{tabular}{|c|c|c|}
\hline Actividad & Objetivo general & $\begin{array}{c}\text { Descripción de la actividad/ } \\
\text { metodología }\end{array}$ \\
\hline $\begin{array}{l}\text { Pósteres informa- } \\
\text { tivos }\end{array}$ & $\begin{array}{l}\text { Consolidar material didáctico de apoyo a los procesos edu- } \\
\text { cativos de enseñanza - aprendizaje de las IE, fortaleciendo } \\
\text { el consumo de frutas y verduras. }\end{array}$ & $\begin{array}{l}\text { Se diseñaron diferentes pósteres con base en } \\
\text { el trabajo adelantado en las IE, a saber: las } \\
\text { frutas; las verduras; buenas prácticas higiéni- } \\
\text { cas en la manipulación de alimentos; huertas } \\
\text { escolares; video resumen de actividades. }\end{array}$ \\
\hline $\begin{array}{l}\text { Guía de prepara- } \\
\text { ciones gastronó- } \\
\text { micas con base en } \\
\text { frutas y verduras }\end{array}$ & $\begin{array}{l}\text { Consolidar con base en las actividades realizadas en el } \\
\text { proceso de sensibilización y capacitación una guía de pre- } \\
\text { paraciones gastronómicas haciendo de las frutas y verduras } \\
\text { el componente principal, además de diferentes actividades } \\
\text { de aprendizaje pedagógicas para incrementar el consumo } \\
\text { de frutas y verduras. }\end{array}$ & $\begin{array}{l}\text { La guía se elaboró teniendo en cuenta los } \\
\text { resultados de las actividades de sensibiliza- } \\
\text { ción y capacitación. }\end{array}$ \\
\hline $\begin{array}{l}\text { Implementación de } \\
\text { la feria de alimen- } \\
\text { tación Sabores y } \\
\text { Saberes }\end{array}$ & $\begin{array}{l}\text { Fomentar en la comunidad académica la importancia de } \\
\text { alimentación saludable, además del consumo y produc- } \\
\text { ción local de alimentos, con énfasis en frutas y verduras. } \\
\text { Socializar los trabajos desarrollados por los escolares con } \\
\text { base en preparaciones con base en frutas y verduras. } \\
\text { Incentivar el intercambio de experiencias y el consumo } \\
\text { de frutas y verduras en todos los estudiantes de la IE Los } \\
\text { Comuneros y el colegio técnico Comfacauca. }\end{array}$ & $\begin{array}{l}\text { La actividad se realizó con la participación } \\
\text { de toda la comunidad educativa, a partir de } \\
\text { la feria de saberes y sabores. Dicho proceso } \\
\text { se desarrollará anualmente. }\end{array}$ \\
\hline
\end{tabular}

Fuente: elaboración propia de los autores.

En síntesis, la metodología puesta en marcha permitió durante esta fase comprender que la integración activa de los miembros de la comunidad académica potencia habilidades comunicativas, destrezas motrices, hábitos alimenticios saludables, junto con el reconocimiento y valoración de las bondades de la diversidad cultural y culinaria de una región como el Cauca, aludiendo a los principios de una educación emancipadora y reflexiva, así como las bases para comenzar a trabajar proyectos educativos institucionales, con preceptos que podría ir por encima de la seguridad alimentaria. 


\section{Conclusiones}

La integración y participación de la comunidad académica de la IE Los Comuneros y el colegio técnico Comfacauca permitieron develar las condiciones bajo las cuales operan sus prácticas alimentarias, así como incrementar el reconocimiento y el fomento del consumo de frutas y verduras locales, y de amplio reconocimiento tradicional y ancestral, sumado a la problematización de los conceptos de seguridad, soberanía alimentaria y territorio dispuestas en las políticas públicas.

Los cambios en los estilos de vida afectan notablemente a la población infantil. En la escuela por lo regular se incrementa el consumo de alimentos altamente energéticos acrecentando los índices de inseguridad alimentaria y con ello el riesgo de sufrir de alguna de las enfermedades crónicas no transmisibles. Aprovechando que la escuela es un ambiente ideal para generar cambios en conductas este espacio se debe aprovechar para enfocar a los niños en la necesidad de estilos de vida saludables, que permitan el mayor consumo de frutas y verduras ya que estos alimentos son ricos en una gran variedad de macro y micronutrientes, además de sustancias biológicamente activas que pueden ser importantes para la prevención de las enfermedades crónicas no transmisibles.

Los niños y las niñas manifiestan que las preparaciones de los alimentos que consumen regularmente son llevadas a cabo por la persona que en ese momento se ocupe de las tareas del hogar, en la mayoría de los casos esta actividad la realizan los padres-madres, seguido de los abuelos(as), y en muchas ocasiones es compartida con ellos, especialmente en el desayuno y el almuerzo; pero es preocupante que no expresan mayor detalle acerca de la comida o cena, porque en un alto porcentaje de los estudiantes de la IE Los Comuneros, no cuentan con alimentos suficientes durante el día, su alimentación principal se concentra en el almuerzo, y en los alimentos del restaurante escolar.

Estas dificultades son similares a la del resto de los niños en el Cauca, y en algunas regiones de Colombia, siendo una apuesta desde la articulación de la educación y la práctica culinaria lograr con investigaciones de esta naturaleza reconocer la riqueza de los alimentos propios, retomarlos e incorporarlos en las dietas de los escolares y sus familias. Con esto se busca impactar en una mejor elección de los alimentos que se consumen regularmente, $y$ en aportar desde la culinaria con recetas nutritivas, deliciosas y a bajo costo.

En la comprensión del nuevo esquema dietario, la escuela deberá abordar el paradigma utilizado por las ciencias de la salud denominado determinantes sociales, el cual ofrece un análisis de contexto que determina los comportamientos humanos derivados de las circunstancias biológicas, sociales, económicas, culturales, geográficas de los seres humanos que nacen, crecen, viven, trabajan y envejecen en determinados "espacios-tiempos". Dicho modelo de análisis adicionalmente ofrece abordar las determinantes biológicas de dicho contexto: biología humana, medioambiente, estilos de vida y conductas de salud y acceso a sistemas de asistencia sanitaria, sumado a los determinantes estructurales en salud: políticas sociales, cultura y valores sociales, políticas públicas, aspectos individuales, costumbres y tradiciones y acceso a la oferta laboral.

De acuerdo con estos preceptos, las dinámicas surgidas en relación con el análisis de las prácticas en materia de soberanía alimentaria y gestión de procesos asociados a ellas se constituyen en sí mismas en insumos articuladores de los retos que plantea el departamento en materia de biodiversidad y servicios ecosistémicos. Lo anterior, sumado a la generación de elementos para el diseño de una política alimentaria que prevea la heterogeneidad de la población, es visto como un pretexto para posicionar al Cauca como un territorio con características diferenciadoras, en torno a las prácticas culinarias tradicionales y ancestrales $y$, por ende, a la gestión de su patrimonio biocultural como herramienta simbólica e inmaterial, acciones susceptibles de conducir a la sustentabilidad, sentando los precedentes para hablar de soberanía alimentaria, entendiendo el acervo cultural como un elemento vivo que dinamiza comunidades y reafirma identidades. 


\section{Agradecimientos}

- IE Los Comuneros, Popayán.

- Colegio técnico Comfacauca, Popayán.

- Universidad Politécnica de Madrid: Ph.D. Virginia Díaz Barcos.

- Innovacción Cauca y Unicomfacauca Popayán (Cauca).

\section{Referencias}

Bock, P. K. (1977). Introducción a la moderna antropología cultural. Madrid: Fondo de Cultura Económica

Crujeriras, A. B., Goyenechea, E. y Martínez, J. A. (2010). Fruit, Vegetables and Legumes Consumption: Role in Preventing and Treating Obesity. España: Department of Nutrition and Food Sciences, Physiology and Toxicology, University of Navarra.

Corporación Autónoma Regional del Cauca (2009). Análisis socioambiental del departamento del Cauca. Popayán: CRC. Documentos públicos.

Delgado, M. y Benavides, E. (2012). Consumo de frutas y verduras en escolares como estrategia preventiva del sobrepeso y obesidad infantiles. Duazary, 9(2), 151-158. Recuperado de http://revistas.unimagdalena.edu.co/index.php/ duazary/article/view/176

Ministerio de Salud y Protección Social (2015). Encuesta Nacional de Situación Nutricional de Colombia. Bogotá. Documentos públicos. Recuperado de https://www.minsalud.gov.co/ salud/publica/epidemiologia/Paginas/encuesta-nacional-de-situacion-nutricional-ensin.aspx

Hernández, R., Fernández, C. y Baptista, P. (2006). Metodología de la investigación. Bogotá: McGraw Hill.

FAO (2015). El estado de la inseguridad alimentaria en el mundo. Roma: FAO.
Jodelet, D. (1989). Représentations socials: un domaine en expansion. En D. Jodelet (ed.), Les représentations sociales. París: PUF.

Lowenberg, M., Todhunter, E., Wilson, E., Feeney, M. y Savage, J. (1970). Los alimentos y el hombre. México D. F.: Limusa-Wiley.

Moscovici, S. (1979). El psicoanálisis, su imagen y su público. Buenos Aires: Huemul.

Secretaría de Salud Departamental del Cauca (2017). Cauca sin hambre, un compromiso del gobierno departamental. Recuperado de https:// saludcauca.gov.co/index.php/sala-de-prensa/ noticias/470-cauca-sin-hambre-un-compromiso-del-gobierno-departamental

Organización Mundial de la Salud y Organización de las Naciones Unidas para la Alimentación y la Agricultura (2007). Diet, Nutrition and the Prevention of Chronic Diseases. OMS: Serie de Informes Técnicos, WHO Technical Report Series 916. Recuperado de https://www.who.int/ dietphysicalactivity/publications/trs916/en/

Muñoz, S. A. (2004). La influencia de la "Nueva Televisión", en las emociones y en la educación de los niños. Revista de Psiquiatría y Psicología del Niño y del Adolescente, 4(2), 145-159.

Pennington, J. y Fisher, R. (2009). Classification of fruits and vegetables. Journal of Food Composition and Analysis, 22, 23-31. DOI: https://doi. org/10.1016/j.jfca.2008.11.012

Piaget, J. (1972). El nacimiento de la inteligencia en el niño. Madrid: Aguilar.

Rodríguez, A. y Rodríguez, S. (2017). Imaginarios y agenciamientos que configuran el consumo de alimentos en escolares. Popayán: Editorial Universidad del Cauca - Unicomfacauca.

Savater, F. (1997). El valor de educar. Barcelona: Ariel. 